\title{
PESQUISA OPERACIONAL UTILIZADA NA MAXIMIZAÇÃO DO PROCESSO DE FABRICAÇÃO DE DISCOS DE ALGODÃO EM UMA EMPRESA DO RAMO DE ALGODÃO HIDRÓFILO
}

\author{
OPERATIONAL RESEARCH USED IN MAXIMIZING THE MANUFACTURING \\ PROCESS OF COTTON DISCS IN A HYDROPHILIC COTTON COMPANY
}

Ciro Ávila Machado Ferreira ${ }^{1}$, Filipe de Oliveira Proença², Guilherme Reis Portilho ${ }^{3 *}$, Victor Hugo Silva Lucas $^{4}$, Zarur de Oliveira Silvano ${ }^{5}$

\footnotetext{
${ }^{1}$ Graduando em Engenharia de Produção das Faculdades Integradas de Cataguases - Grupo Unis, Cataguases, MG, Brasil, ciro.ferreira@alunos.unis.edu.br

${ }^{2}$ Graduando em Engenharia de Produção das Faculdades Integradas de Cataguases - Grupo Unis, Cataguases, MG, Brasil, filipe.proenca@alunos.unis.edu.br

${ }^{3}$ Graduando em Engenharia de Produção das Faculdades Integradas de Cataguases - Grupo Unis, Cataguases, MG, Brasil, guilherme.portilho@alunos.unis.edu.br

${ }^{4}$ Graduando em Engenharia de Produção das Faculdades Integradas de Cataguases - Grupo Unis, Cataguases, MG, Brasil, victor.lucas@alunos.unis.edu.br

${ }_{5}^{5}$ Professor nas Faculdades Integradas de Cataguases - Grupo Unis, Cataguases, MG, Brasil, zarurs@yahoo.com.br
}

\begin{abstract}
Resumo
A pesquisa operacional $(\mathrm{PO})$ é um instrumento de tomada de decisão muito respeitável devido a sua forma lógica de decorrer. $\mathrm{O}$ alvo é mostrar a seriedade da PO para a tomada racional de decisão, como usar o recurso Solver no Excel, e como chegar ao saldo de maximização em apontado processo de fabricação de discos de algodão em uma empresa do ramo de algodão hidrófilo. Foi utilizado diferentes bibliografias para o alicerce teórico do trabalho, também utilizou-se de cronometragem de processos na empresa e o programa Microsoft Office Excel para a resolução do problema de maximização do processo. Como resultado, notou-se que todos os processos estão atingindo o seu limite de caixas. Essa realidade demonstra a necessidade de um estudo direcionado e focado na otimização e maximização da produção do item Disco.
\end{abstract}

Palavras-Chave: Programação Linear, Pesquisa Operacional, Solver.

\begin{abstract}
Operations research (OP) is a very respectable decision-making tool due to its logical way of proceeding. The aim is to show the seriousness of the PO for rational decision making, how to use the Solver feature in Excel, and how to reach the balance of maximization in a pointed cotton disk manufacturing process in a company in the cotton field. Different bibliographies were used for the theoretical foundation of the work, we also used process timing in the company and the Microsoft Office Excel program to solve the process maximization problem. As a result, it was noticed that all processes are reaching their box limit. This reality demonstrates the need for a focused study focused on optimizing and maximizing the production of the Disc item.
\end{abstract}

Keyword (s): Linear Programming, Operational Research, Solver.

(C)NIS-MG. All rights reserved. 


\section{INTRODUÇÃO}

Segundo CONAB, ABRAPA (2018), nos últimos anos, o Brasil tem se mantido entre os cinco maiores produtores mundiais de algodão, ao lado de países como China, Índia, EUA e Paquistão. Ocupa o primeiro lugar em produtividade em sequeiro. O Brasil tem figurado também entre os maiores exportadores mundiais. O cenário interno é promissor, pois o Brasil está entre os maiores consumidores mundiais de algodão em pluma.

De acordo com Jacobs (2009), o segredo das produções lucrativas é fazer o melhor uso dos recursos disponíveis: profissionais, materiais, estrutura fabril, equipamentos e dinheiro. O gerente moderno dispõe de uma ferramenta de modelagem matemática eficiente para essa finalidade, por meio da programação linear.

Para o desenvolvimento e otimização do mix de produção de uma indústria, objetivando a maximização de seu ganho, as empresas precisam de ferramentas ágeis que tragam resultados precisos e analisem as variáveis e as exceções do sistema produtivo e de mercado.

O presente trabalho tem como objetivo geral proporcionar a pesquisa operacional como uma ferramenta coerente em tomada de decisão em uma empresa do ramo de Algodão. Cuja finalidade é a produção de algodão Hidrófilo sendo eles os produtos: $25 \mathrm{~g}, 50 \mathrm{~g}, 100 \mathrm{~g}, 250 \mathrm{~g}, 500 \mathrm{~g}$, Bola, Disco. Desta maneira procura-se melhorar a otimização do mix de produção de Disco. Com os dados adquiridos através de visitas obtendo assim cronometragens, foi feito uma análise de tempo real e sintético.

Os objetivos principais são estudar e analisar a produção de disco de algodão, e mostrar como utilizar o Solver do Microsoft office Excel, bem como achar a solução que maximize o tempo de produção desse processo. Portanto o Disco foi escolhido para ser analisado e estudado.

\section{FUNDAMENTAÇÃO TEÓRICA}

\subsection{Pesquisa operacional}

De Acordo com Hillier (2013), a pesquisa operacional envolve pesquisa sobre operações". Portanto a PO é aplicada a problemas que compreendem a condução e a coordenação das operações (as atividades) em uma organização, A natureza das organizações é essencialmente secundária e, de fato, a PO tem sido amplamente aplicada em áreas tão distintas como, manufatura, transportes, construção, telecomunicações, planejamento financeiro, assistência médica, militar e serviços públicos. Portanto a gama de aplicações é excepcionalmente grande.

Segundo Fávero (2012), é crescente a necessidade de utilização das ferramentas da Pesquisa Operacional em um período recente em função de alguns aspectos: Aprimoramento das técnicas de pesquisa e de levantamento de dados, que possibilitam a geração de bases com relevância amostral; entendimento por parte dos pesquisadores, das mais diversas áreas de estudo; desenvolvimento de pacotes computacionais que permitem a inclusão de uma quantidade enorme de dados e possibilitam a elaboração de modelos com maior rapidez e precisão.

\subsection{Programação Linear}

De acordo com Loeach, et al. (2009), a programação linear é a resolução de maximização (como lucro) ou minimização (como custo) de algum objetivo, atendendo a um conjunto de 
FERREIRA, Ciro; PROENÇA, Filipe. PORTILHO, Guilherme; LUCAS, Victor; SILVANO, Zarur. Pesquisa operacional utilizada na maximização do processo de fabricação de discos de algodão em uma empresa do ramo de algodão hidrófilo.

restrições. Parte da modelagem do problema culmina na obtenção da solução ótima. As variáveis são reais (isto é, números são necessariamente inteiros).

Segundo Paulo (2013), os resultados positivos alcançados pelo grupo de cientistas ingleses fizeram com que a pesquisa operacional fosse disseminada nos Estados Unidos e, em 1947, a equipe liderada por George B. Dantzig deu origem ao método Simplex para resolução de problemas de Programação Linear, desde então esse conhecimento vem sendo aplicado, com sucesso, para a otimização de recursos em diversos segmentos industriais e comerciais de várias áreas de negócio.

\subsection{Fluxograma}

Segundo Lócio (2010), o fluxograma é a representação gráfica para descrever atividades realizadas em empresas. Os símbolos básicos geométricos que colocam em evidenciar o início do processo podem ser, círculos, losango, retângulos, linhas ou setas. Geralmente, usa-se um círculo alongado para diferenciar o início e o fim do fluxo; o losango retrata questões / alternativas (o losango sempre terá duas saídas); no retângulo são colocadas as ações; a seta é utilizada para dar sentido do fluxo; as linhas ou setas nunca devem cruzar umas sobre as outras; o texto deve ser sempre claro e sucinto.

De acordo com Oliveira (2009), as principais vantagens de um fluxograma é: facilitar a leitura e o conhecimento, facilitar o local e a identificação dos fatos mais importantes, maior agilidade e melhor grau de análise, clareza na descrição dos métodos administrativos, uniformizar o conceito dos métodos e os processos administrativos.

\subsection{Tempo e Movimento}

O estudo de tempo e movimento tem como objetivo, segundo DUARTE (2015):

“(1) estabelecer requisitos e padrões do cargo ou da função; (2) levantamento e determinação precisos da quantidade necessária de tempo a partir da avaliação comparativa em relação em relação ao padrão anteriormente, estabelecido da capacidade ou do esforço realizado pelo trabalhador objetivando a execução de uma tarefa específica; (3) fundamenta-se na análise e na medida de todos os tempos executados num trabalho, visando eliminar os esforços desnecessários." (DUARTE,2015)

O desenvolvimento do método preferido ou ideal parte primeiramente da definição de um problema, onde são criados relatórios com base nas metas e objetivos existentes. Consequentemente será realizado a análise de problemas descrevendo todos os métodos incluindo especificações e restrições de produção. Desta forma, com o surgimento de possíveis soluções, são realizadas avaliações objetivando o método que proporciona menor custo e assim menor investimento, contribuindo para um processo de produção mais rápido e eficaz. Com a utilização desta metodologia torna-se possível processar um sequenciamento de operações que se aproximam do "ideal".

Após a análise e a obtenção de um novo método para operações de produção, deve ser padronizado. A tarefa passa a ser dividida entre em trabalhos ou operações especificas.

Segundo Figueiredo, Oliveira, Santos (2011), padronizada a operação o passo seguinte será a determinação do tempo padrão, o mesmo visa identificar o tempo que o operador qualificado e treinado deveria gastar para executar certa operação. Com a coleta destes dados torna-se possível fazer um planejamento e uma programação a qual estimara o custo da mão-de-obra a ser utilizada, ferramenta base para incentivos salariais e ainda como planejamento e controle da 
produção. E para finalizar o estudo dos tempos e de movimentos o treinamento do operador é de grande relevância para que o operador possa executar suas operações sem maiores dificuldades.

De acordo com Picanco, et al. (2011), a padronização das operações e o conhecimento acurado da capacidade produtiva têm influência direta na eficácia do PCP (Planejamento e Controle da Produção) pelo alcance de alguns de seus objetivos como: redução dos lead times de produção, possibilidade de cumprimento de prazos e agilidade de resposta diante de alterações de demanda.

\section{METODOLOGIA}

De acordo Tardin et al (2013) apud Gil (1999), a metodologia é uma série de raciocínio. Foram realizadas pesquisas Bibliográficas por meio de livros e internet, também foram consultados artigos científicos. Todas as buscas foram efetuadas no período, entre 07 de março a 01 de junho de 2020. A escolha dos Artigos foi feita em semelhança ao tema proposto. Para a realização do estudo de caso foram feitas medições na empresa estudada, em que o processo analisado foi a produção do Algodão Disco. Foram cronometrados tempo do processo e o movimento dos operadores também foram estudados.

Diante dos dados coletados e observação do processo foi obtida uma base de dados para ser trabalhada. Sendo assim, a construção de fluxograma para melhor visualização do processo. A resolução desse trabalho deu-se através da ferramenta Microsoft office Excel 365, foi utilizado também uma ferramenta que está dentro do Excel chamada Solver. A construção do fluxograma foi feita através do software Bizage.

\subsection{EMPRESA OBJETO DE ESTUDO}

A análise foi realizada em uma empresa de Médio Porte, sua produção tem como seguimento de Algodão Hidrófilo. Localizada na Zona da Mata, na cidade Cataguases, Minas Gerais, Brasil. A empresa trabalha com diversas linhas de produtos e os comercializa para o todo o território nacional.

\section{RESULTADOS E DISCUSSÕES}

Diante dos conceitos supracitados e do estudo in loco, criou-se o modelo de fluxograma do processo de produção dos discos de algodão, desde o momento de recebimento da matéria-prima até o armazenamento no estoque (figura 1). 
FERREIRA, Ciro; PROENÇA, Filipe. PORTILHO, Guilherme; LUCAS, Victor; SILVANO, Zarur. Pesquisa operacional utilizada na maximização do processo de fabricação de discos de algodão em uma empresa do ramo de algodão hidrófilo.

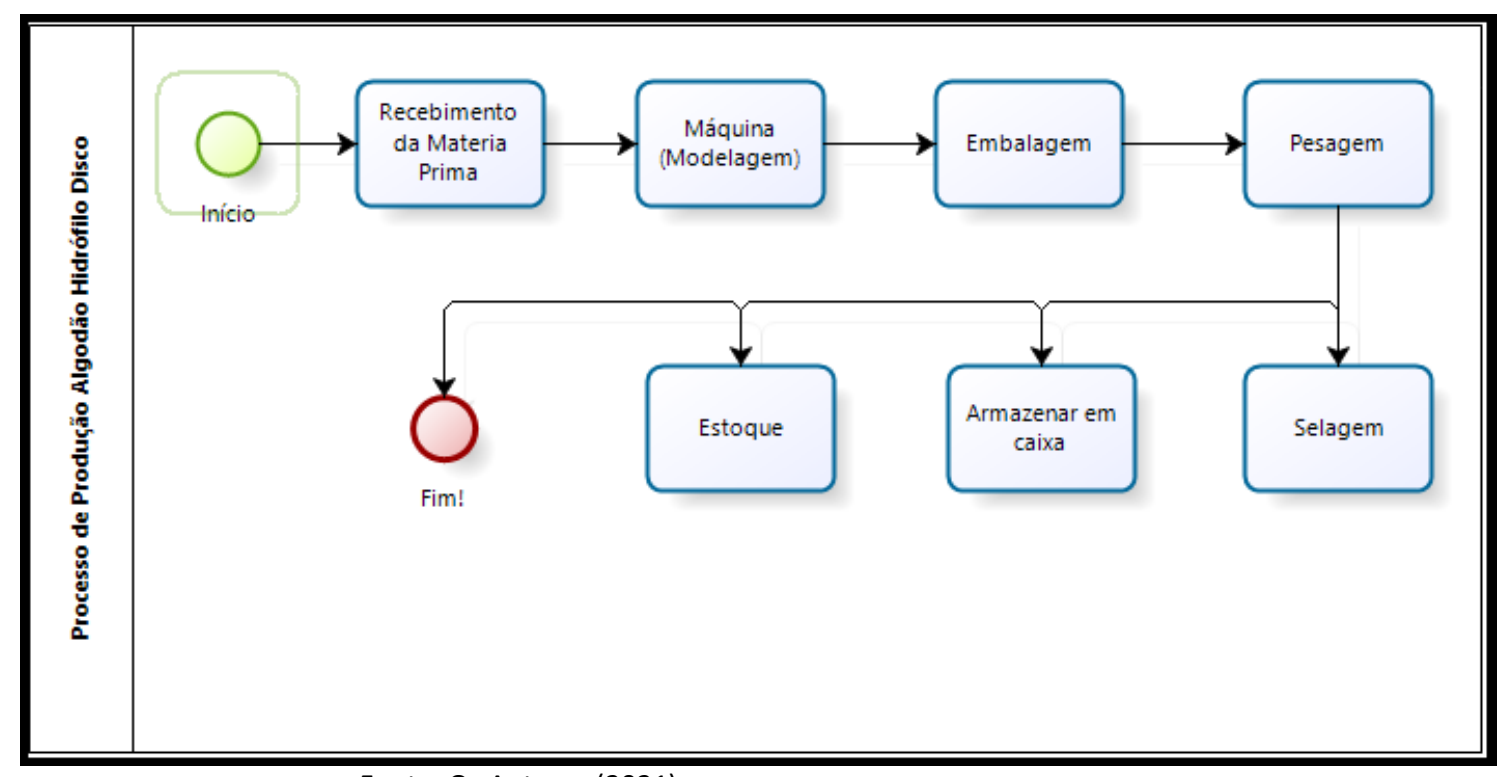

Fonte: Os Autores (2021)

Figura 1 - Fluxograma

Na primeira etapa acontece o recebimento da matéria-prima (algodão alvejado), no processo de modelagem a máquina modela o material no formato de uma manta que será prensada em várias unidades que serão embaladas, pesadas, seladas e armazenadas em caixas de acordo com o tipo de produto final. Ao término do processo, as caixas seguem para armazenamento no estoque.

A fim de analisar o tempo de produção de uma caixa de cada produto da empresa, os processos foram cronometrados e agrupados em uma tabela como demonstra a figura 2.

\begin{tabular}{|c|c|c|c|c|c|c|c|}
\hline \multicolumn{7}{|c|}{ Algodão Hidrófilo } \\
\hline $500 \mathrm{~g}$ & $250 \mathrm{~g}$ & $25 \mathrm{~g}$ P/Unidade & $25 \mathrm{~g}$ KG & $100 \mathrm{~g}$ P/Unidade & $100 \mathrm{~g}$ KG & Disco & Disco \\
\hline $00: 00: 40$ & $00: 01: 00$ & $00: 00: 02$ & $00: 01: 35$ & $00: 00: 05$ & $00: 00: 27$ & $6 \mathrm{cxs} \mathrm{p} / \mathrm{hr}$ & $00: 09: 47$ \\
\hline $00: 00: 45$ & $00: 00: 59$ & $00: 00: 05$ & $00: 01: 33$ & $00: 00: 08$ & $00: 00: 20$ & $6 \mathrm{cxs} \mathrm{p} / \mathrm{hr}$ & $00: 10: 15$ \\
\hline $00: 00: 42$ & $00: 01: 04$ & $00: 00: 04$ & $00: 01: 24$ & $00: 00: 07$ & $00: 00: 23$ & $6 \mathrm{cxs} \mathrm{p} / \mathrm{hr}$ & $00: 09: 57$ \\
\hline $00: 00: 40$ & $00: 00: 58$ & $00: 00: 03$ & $00: 01: 16$ & $00: 00: 06$ & $00: 00: 22$ & $6 \mathrm{cxs} \mathrm{p} / \mathrm{hr}$ & $00: 10: 23$ \\
\hline $00: 00: 46$ & $00: 01: 05$ & $00: 00: 04$ & $00: 01: 29$ & $00: 00: 08$ & $00: 00: 21$ & $6 \mathrm{cxs} \mathrm{p} / \mathrm{hr}$ & $00: 09: 15$ \\
\hline $00: 00: 41$ & $00: 00: 55$ & $00: 00: 06$ & $00: 01: 15$ & $00: 00: 10$ & $00: 00: 30$ & $6 \mathrm{cxs} \mathrm{p} / \mathrm{hr}$ & $00: 10: 18$ \\
\hline $00: 00: 40$ & $00: 00: 56$ & $00: 00: 02$ & $00: 01: 22$ & $00: 00: 05$ & $00: 00: 26$ & $6 \mathrm{cxs} \mathrm{p} / \mathrm{hr}$ & $00: 10: 05$ \\
\hline $00: 00: 42$ & $00: 00: 54$ & $00: 00: 03$ & $00: 01: 33$ & $00: 00: 06$ & $00: 00: 27$ & $6 \mathrm{cxs} \mathrm{p} / \mathrm{hr}$ & $00: 09: 36$ \\
\hline $00: 00: 40$ & $00: 01: 15$ & $00: 00: 04$ & $00: 01: 25$ & $00: 00: 07$ & $00: 00: 24$ & $6 \mathrm{cxs} \mathrm{p} / \mathrm{hr}$ & $00: 10: 00$ \\
\hline $00: 00: 49$ & $00: 00: 58$ & $00: 00: 06$ & $00: 01: 27$ & $00: 00: 09$ & $00: 00: 19$ & $6 \mathrm{cxs} \mathrm{p} / \mathrm{hr}$ & $00: 09: 15$ \\
\hline $00: 00: 42$ & $00: 00: 59$ & $00: 00: 04$ & $00: 01: 30$ & $00: 00: 07$ & $00: 00: 20$ & $6 \mathrm{cxs} \mathrm{p} / \mathrm{hr}$ & $00: 09: 55$ \\
\hline $00: 00: 43$ & $00: 00: 53$ & $00: 00: 06$ & $00: 01: 28$ & $00: 00: 06$ & $00: 00: 21$ & $6 \mathrm{cxs} \mathrm{p} / \mathrm{hr}$ & $00: 10: 10$ \\
\hline $00: 00: 52$ & $00: 00: 58$ & $00: 00: 04$ & $00: 01: 38$ & $00: 00: 07$ & $00: 00: 18$ & $6 \mathrm{cxs} \mathrm{p} / \mathrm{hr}$ & $00: 09: 42$ \\
\hline $00: 00: 40$ & $00: 01: 10$ & $00: 00: 05$ & $00: 01: 21$ & $00: 00: 06$ & $00: 00: 25$ & $6 \mathrm{cxs} \mathrm{p} / \mathrm{hr}$ & $00: 09: 24$ \\
\hline $00: 00: 43$ & $00: 00: 55$ & $00: 00: 02$ & $00: 01: 30$ & $00: 00: 06$ & $00: 00: 28$ & $6 \mathrm{cxs} \mathrm{p} / \mathrm{hr}$ & $00: 10: 05$ \\
\hline $00: 00: 41$ & $00: 01: 13$ & $00: 00: 03$ & $00: 01: 24$ & $00: 00: 07$ & $00: 00: 18$ & $6 \mathrm{cxs} \mathrm{p} / \mathrm{hr}$ & $00: 10: 12$ \\
\hline
\end{tabular}

Figura 2 - Cronometragem dos Processos

$\mathrm{Na}$ Figura 2, foram selecionados sete produtos comercializados pela empresa e foram feitas dezesseis cronometragens de todo o processo de produção de cada um, para assim poder ter um melhor entendimento e saber com qual dos processos/produto trabalhar. Após essa análise, foi 
FERREIRA, Ciro; PROENÇA, Filipe. PORTILHO, Guilherme; LUCAS, Victor; SILVANO, Zarur. Pesquisa operacional utilizada na maximização do processo de fabricação de discos de algodão em uma empresa do ramo de algodão hidrófilo.

selecionado o Disco que é apresentado nas duas últimas colunas da tabela. A primeira apresentando a quantidade de caixas produzidas por hora e a segunda as dezesseis cronometragens dessa linha de produção.

\begin{tabular}{|c|c|c|}
\hline \multicolumn{3}{|c|}{ Disco } \\
\hline Dias & Tempo & Caixas \\
\hline Segunda & 10 & 6 \\
\hline Terça & 9 & 6 \\
\hline Quarta & 10 & 7 \\
\hline Quinta & 10 & 6 \\
\hline Sexta & 9 & 8 \\
\hline Sábado & 9 & 6 \\
\hline
\end{tabular}

Fonte: Os Autores (2021)

Figura 3 - Processo do Disco cronometrado em uma semana de trabalho

A figura 3 mostra o processo realizado em uma semana de trabalho de produção do item disco de algodão com o total de caixas produzidas em cada dia da semana.

As variáveis de decisão representam cada processo realizado em uma semana de trabalho, sendo cronometrados apenas alguns processos por hora.

X1=Número de Caixas produzidas na Segunda

X2=Número de Caixas produzidas na Terça

X3=Número de Caixas produzidas na Quarta

X4=Número de Caixas produzidas na quinta

X5=Número de Caixas Produzidas na Sexta

X6=Número de Caixas produzidas no Sábado.

O objetivo do problema é maximizar a produção do Disco, através das variáveis citadas anteriormente. Descobrindo qual a melhor distribuição de processos por tempo que maximize a produção do Disco em um determinado período, como mostra a Figura 4:

\begin{tabular}{|c|c|}
\hline \multicolumn{2}{|c|}{ Disco } \\
\hline Dias & Tempo \\
\hline Segunda & 10 \\
\hline Terça & 9 \\
\hline Quarta & 10 \\
\hline Quinta & 10 \\
\hline Sexta & 9 \\
\hline Sábado & 9 \\
\hline
\end{tabular}

Fonte: Os Autores (2021)

Figura 4 - Dias da Semana por tempo

A soma dos dias da semana, multiplicada pelo tempo que realizam os processos irá resultar em uma elevada produção de caixa em um determinado tempo. Para isso segue a função que irá maximizar. 
FERREIRA, Ciro; PROENÇA, Filipe. PORTILHO, Guilherme; LUCAS, Victor; SILVANO, Zarur. Pesquisa operacional utilizada na maximização do processo de fabricação de discos de algodão em uma empresa do ramo de algodão hidrófilo.

$$
\operatorname{MaxZ}=10 \times 1+9 \times 2+10 \times 3+10 \times 4+9 \times 5+9 \times 6
$$

\subsection{Restrições da Função}

1) A primeira restrição é relacionada a quantidade máxima de caixas produzidas em um determinado período da semana.

$$
\mathrm{X} 1+\mathrm{X} 2+\mathrm{X} 3+\mathrm{X} 4+\mathrm{X} 5+\mathrm{X} 6 \leq 39
$$

2) Segunda restrição é a quantidade de caixas produzidas na segunda feira.

$$
\mathrm{X} 1 \leq 6
$$

3) Terceira restrição é a quantidade de caixas produzidas na terça feira.

$$
\mathrm{X} 2 \leq 6
$$

4) Quarta restrição é a quantidade de caixas produzidas na quarta-feira.

$$
\mathrm{X} 3 \leq 7
$$

5) Quinta restrição é a quantidade de caixas produzidas na quinta feira.

$$
\mathrm{X} 4 \leq 6
$$

6) Sexta restrição é a quantidade de caixas produzidas na sexta feira.

$$
\mathrm{X} 5 \leq 8
$$

7) Sétima restrição é a quantidade de caixas produzidas no sábado.

$$
x 6 \leq 6
$$

Sendo assim o problema tem o seguinte aspecto matemático:

MaxZ $=10 \times 1+9 \times 2+10 \times 3+10 \times 4+9 \times 5+9 \times 6$

Sujeito a:

$\mathrm{X} 1+\mathrm{X} 2+\mathrm{X} 3+\mathrm{X} 4+\mathrm{X} 5+\mathrm{X} 6 \leq 39$

$\mathrm{X} 1 \leq 6$

$\mathrm{X} 2 \leq 6$

$\mathrm{X} 3 \leq 7$

$\mathrm{X} 4 \leq 6$

$\mathrm{x} 5 \leq 8$

$\mathrm{x} 6 \leq 6$

$X 1+X 2+X 3+X 4+X 5+X 6 \geq 0$

\subsection{Resolução do Problema Usando o Instrumento Solver do Microsoft Office Excel.}

O software usado para a preparação e solução do problema foi a ferramenta Solver do Excel pois é um software que oferece a maior facilidade de manejo e melhor arranjo dos relatórios gerados pela operação. Para a utilização do Solver, as informações coletadas na empresa foram depositadas no Excel e, dessa maneira, foi descoberto um número ótimo de caixas que maximizará o processo. 
FERREIRA, Ciro; PROENÇA, Filipe. PORTILHO, Guilherme; LUCAS, Victor; SILVANO, Zarur. Pesquisa operacional utilizada na maximização do processo de fabricação de discos de algodão em uma empresa do ramo de algodão hidrófilo.

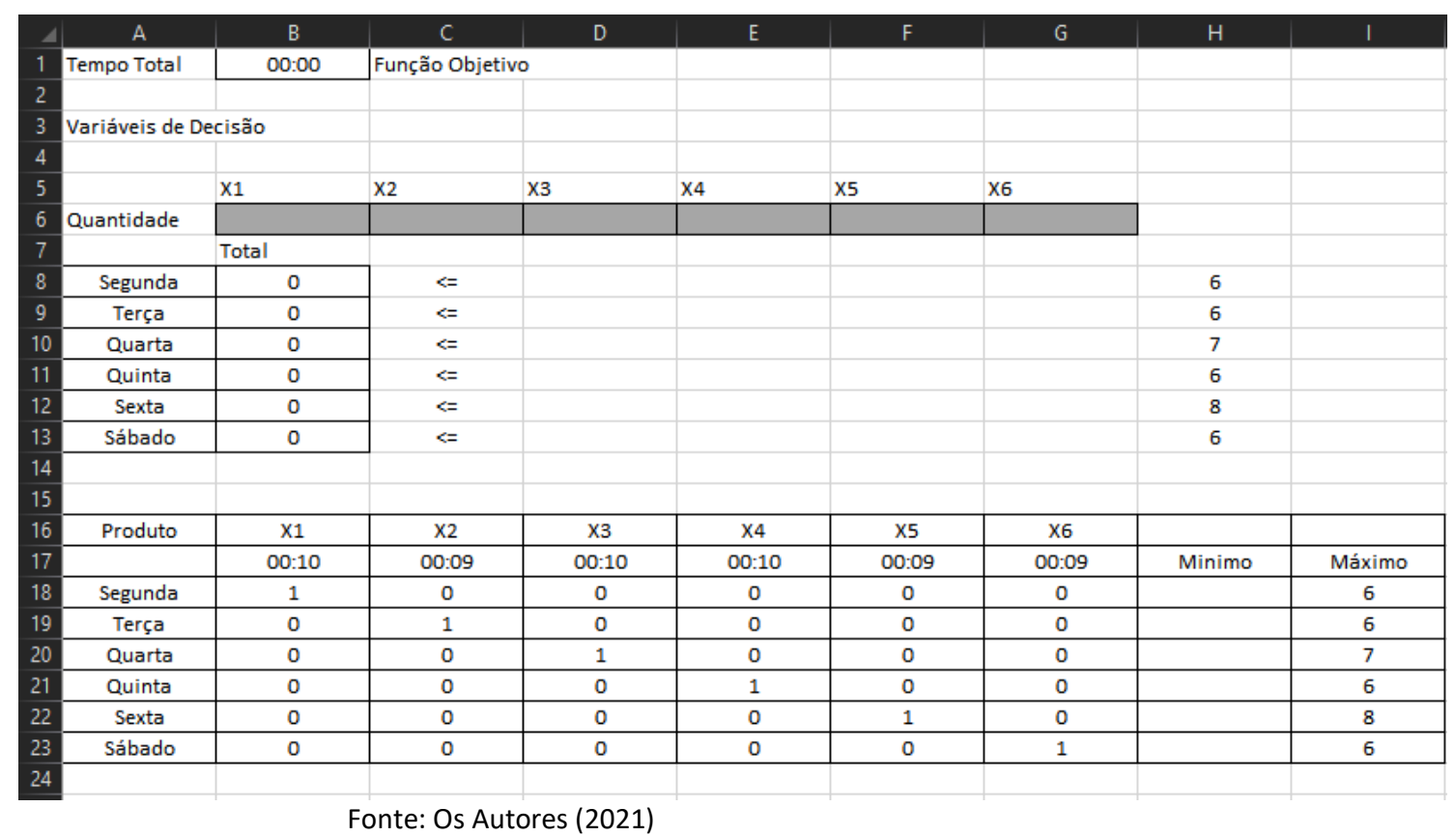

Figura 5 - Dados do Problema no Excel Solver

Observando na Figura 5, na linha 16, tem-se as variáveis de decisão do problema e embaixo de cada uma (linha 17) seus respectivos coeficientes. Mais abaixo encontram-se os coeficientes das restrições das colunas, $B, C, D, E, F, G$, nas linhas 18 a 23. Também na coluna $C$ das linhas 8 a 13 estão os símbolos de menor igual, indicando qual o tipo de restrição. $\mathrm{Na}$ coluna $\mathrm{H}$ (idem as linhas) estão os valores das restrições (ex. 39 total de caixas, 6 caixas na segunda etc.).

Na linha 6 contém a solução do problema, Antes de utilizar o solver, deve-se colocar o valor 0 na solução, para entrada do problema. Deve se observar as fórmulas que estão na B1, B8 a B13.

A fórmula B1, diz respeito ao somatório da solução de cada variável vezes o coeficiente da mesma (Total de Receitas). Sendo assim, segue a fórmula:

=SOMARPRODUTO(B6:G6;B17:G17)

Já as fórmulas B8 A B1 diz respeito a solução de cada variável vezes o coeficiente restritivo da mesma, ou seja, as fórmulas B8 a B13 são:

=SOMARPRODUTO(B6:G6;B18:G18)

=SOMARPRODUTO(B6:G6;B19:G19)

=SOMARPRODUTO(B6:G6;B20:G20)

$=$ SOMARPRODUTO(B6:G6;B21:G21)

=SOMARPRODUTO(B6:G6;B22:G22)

=SOMARPRODUTO(B6:G6;B23:G23)

Observe que os campos da Solução B6 a G6 se repetem. Para colocar a fórmula no primeiro campo e arrastá-la para valer nos outros campos, deve-se selecionar o campo que se repete e clicar F4, para que se mantenha fixo, ao arrastar a fórmula. Caso a ferramenta Solver não esteja habilitada no Excel vá na aba ferramentas, Suplementos e marque a Opção Solver.

Depois de ter obtido todos os dados, e jogado em seu devido lugar na planilha vá em Dados, Solver. Abrirá a seguinte caixa de diálogo. 
FERREIRA, Ciro; PROENÇA, Filipe. PORTILHO, Guilherme; LUCAS, Victor; SILVANO, Zarur. Pesquisa operacional utilizada na maximização do processo de fabricação de discos de algodão em uma empresa do ramo de algodão hidrófilo.

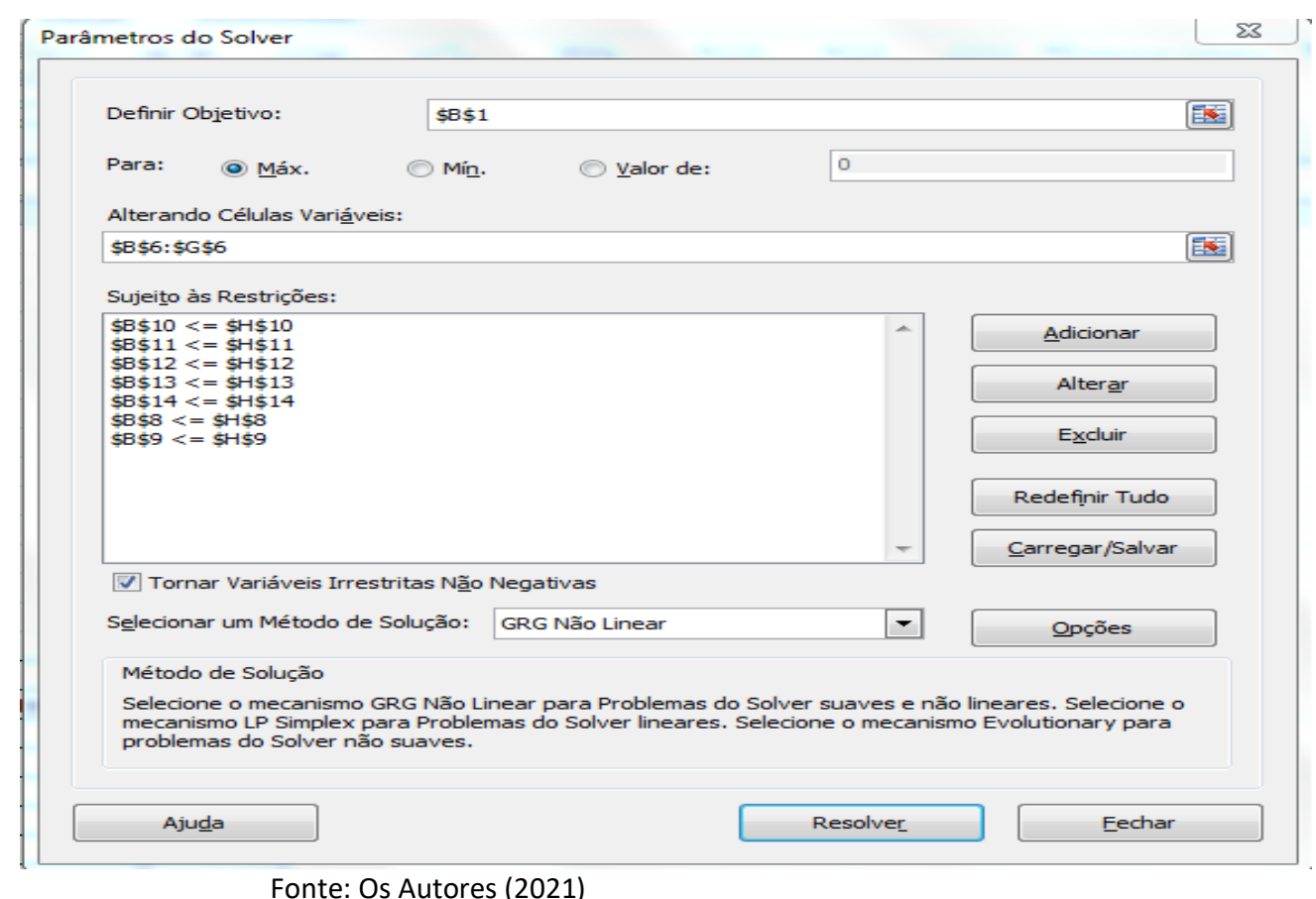

Figura 6 - Caixa de Diálogo Solver

No Campo "Definir Objetivo" clique no campo B1, que contém o somatório da solução de cada variável vezes o coeficiente da mesma. (Custo Total).

No item "igual a", marque a opção de Máx, que índica um problema de maximização neste caso.

Vá no Botão Adicionar, para selecionar as restrições. Observe que foi alocado: o campo das fórmulas da coluna B8 a B13 uma de cada vez com o símbolo menor igual e o campo onde estão os limites (C8 a C13). Em seguida de Ok. Após, o Excel apresentará o resultado com a solução do problema (conforme figura 8).

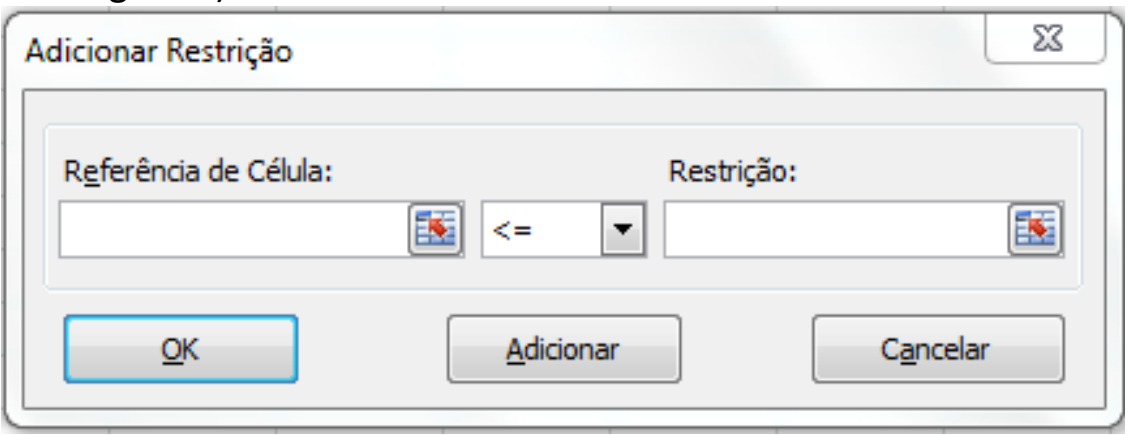

Fonte: Os Autores (2021)

Figura 7 - Adição de restrição do problema 
FERREIRA, Ciro; PROENÇA, Filipe. PORTILHO, Guilherme; LUCAS, Victor; SILVANO, Zarur. Pesquisa operacional utilizada na maximização do processo de fabricação de discos de algodão em uma empresa do ramo de algodão hidrófilo.

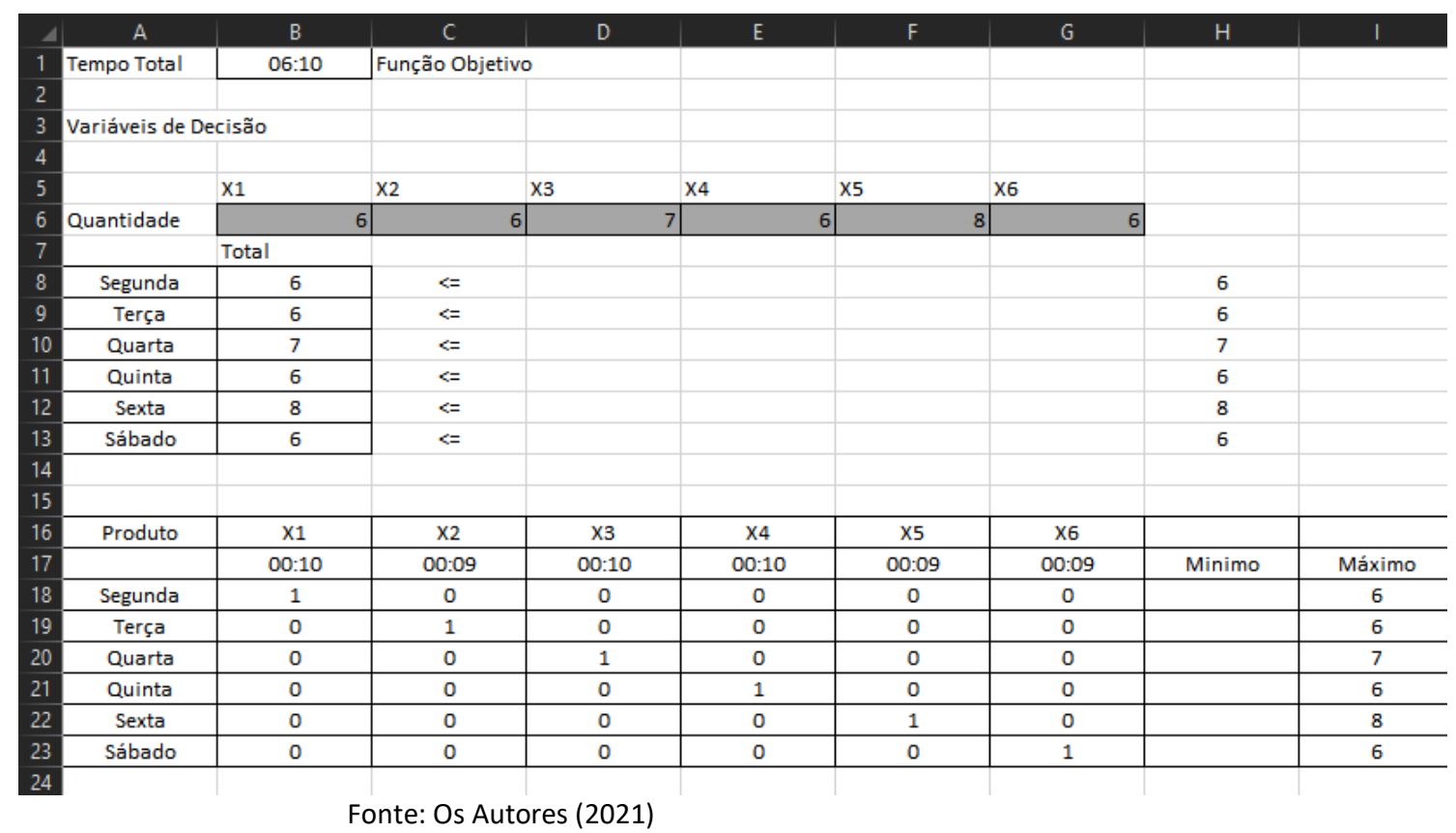

Figura 8 - Janela do Excel com a Solução do Problema

Essas quantidades de caixas produzidas representam um tempo total de $6 \mathrm{~h}$ e $10 \mathrm{~min}$ (Seis horas e dez Minutos) em um determinado processo em unidades por caixa. Sobra do tempo disponível de produção (7 horas) o total de 50 minutos, este tempo representa uma perda diária de produção no qual pode ser tratada posteriormente.

\section{CONSIDERAÇÕES FINAIS}

O estudo da PO, levando em conta a sua sistemática leva o técnico a adquirir um raciocínio estruturado. Essa formalização do raciocínio proporciona rápida análise e interpretação dos problemas reais, proporcionando a um monitoramento detalhado dos aspectos envolvidos. No entanto, como o técnico de PO na maior parte das vezes não é um profundo especialista da área em que será aplicado o modelo, é fundamental um relacionamento constante com o usuário, principalmente nas etapas iniciais de formulação modelagem. Esse relacionamento se torna ainda mais relevante à medida que o usuário precisa estar convencido da validade e das vantagens que essas técnicas proporcionam, para que esteja garantida a viabilidade de sua utilização. No tocante aos dados aplicados nos modelos, é muito importante que seja conhecida a sua qualidade, pois, às vezes, procura-se refinar um modelo, sem levar em consideração que a qualidade das informações necessárias a esse refinamento não o justifica. Um estudo utilizando a análise de sensibilidade, em muitos casos, possibilita verificar a influência de determinado dado (parâmetro), o que poderia não justificar um melhor detalhamento do modelo.

Um bom planejamento da produção é imprescindível para garantir a qualidade dos produtos comercializados, o cumprimento dos prazos estabelecidos e a otimização e a maximização dos processos. A partir desse ponto, com o objetivo de explorar e exemplificar um desafio enfrentado no dia a dia das indústrias, esse estudo foi realizado por meio de dados cronometrados, que através de algumas análises foi identificada a necessidade de otimizar a produção do Disco de Algodão, pois sua demanda está cada vez maior, gerando assim o maior número de caixas possíveis em menor tempo. Este trabalho foi promovido pelo estudo de tempo e análise dos processos da empresa. 
FERREIRA, Ciro; PROENÇA, Filipe. PORTILHO, Guilherme; LUCAS, Victor; SILVANO, Zarur. Pesquisa operacional utilizada na maximização do processo de fabricação de discos de algodão em uma empresa do ramo de algodão hidrófilo.

Para a obtenção dos dados foi necessário analisar o processo in loco, entender o processo de produção e cruzar as informações com o auxílio do Microsoft Excel. Com uma observação criteriosa e analítica, pode-se observar que todos os processos estão atingindo o seu limite de produção, tanto o X1, X2, X3, X4, X5, X6. Essas quantidades de caixas produzidas representa um tempo total de $6 \mathrm{~h}$ e $10 \mathrm{~min}$ (seis horas e dez minutos) em um determinado processo em unidades por caixa. Esse número precisa ser mais bem explorado, estudado e analisado a fim de melhorá-lo e realmente otimizar e maximizar a produção do item Disco.

De forma genérica percebe-se que o conhecimento de Pesquisa Operacional e o recurso por programação linear, faz com que os processos dentro de uma empresa ganhem uma vantagem pela ferramenta gerencial em seus processos para a tomada de decisão.

\section{REFERÊNCIAS}

ABAPRA Associação Brasileira dos Produtos de Algodão. CONAB 04/12/2018 Disponível em < https://www.abrapa.com.br/Paginas/dados/algodao-no-brasil.aspx> Acesso em 01/12/2018.

FAVERO (2012) apud BELFIORE, Pesquisa Operacional, Disponível em https://books.google.com.br/books?id=spbIUnIPwn8C\&printsec=frontcover\&dq=pesquisa+operac ional\&hl=pt-

$B R \& s a=X \& v e d=0 a h U K E$ wictqblzoTfAhVDgpAKHXS5CnEQ6AEIVDAH\#v=onepage\&q=pesquisa\%20op eracional $\& f=$ false $>$ Acesso em 03/12/2018.

HILLIER (2013) apud LIEBERMAN. Introdução a Pesquisa Operacional. Disponível em< https://books.google.com.br/books?id=-

A88aOKxQ0C\&printsec=frontcover\&dq=pesquisa+operacional\&hl=ptBR\&sa=X\&ved=0ahUKEwjtYKIyYTfAhXBjZAKHRAbBmcQ6AEIKTAA\#v=onepage $\& q=$ pesquisa\%20operacional $\& f=f a l>$ Acesso em 25/11/2018.

JACOBS (2009) apud CHASE. Administração da Produção e de Operações O Essencial. Disponível em<https://books.google.com.br/books?id=qRsQaZ_-

fRwC\&pg=PA392\&dq=programa\%C3\%A7\%C3\%A3o+linear+solver\&hl=pt-

BR\&sa=X\&ved=0ahUKEwisl9_iyoHfAhUMiZAKHeP4BDkQ6AEINTAC\#v=onepage\&q=programa\%C3 \%A7\%C3\%A3o\%20linear\%20solver\&f=false> Acesso 28/11/2018

LÓCIO F. Fluxograma10/04/2010 Disponível em <http://www.scribd.com /doc/36162383/osm1fluxograma $>$ Acesso em 18/10/2018

LOESCH (2009) apud HEIN. Pesquisa Operacional, fundamentos e modelos. Disponível em $<$ https://books.google.com.br/books?id=ZyprDwAAQBAJ\&printsec=frontcover\&dq=pesquisa+oper acional\&hl=pt-BR\&sa=X\&ved=0ahUKEwjt-

YKIyYTfAhXBjZAKHRAbBmcQ6AEIPjAD\#v=onepage\& $q=$ pesquisa\%20operacional $\& f=f a l s>$ Acesso em 18/10/2018.

ROCHA Anselmo Neto, ZANELLA Cleunice. Planejamento da produção com a utilização do SolverExcel na empresa Bondio Alimentos S.A. XXVII Encontro Nacional de Engenharia de Produção Enegep. Disponível em < http://www.pucrs.br/ciencias/viali/graduacao/po_2/literatura/excel_solver/enegep2007.pdf> Acesso em 15/11/2018. 
FERREIRA, Ciro; PROENÇA, Filipe. PORTILHO, Guilherme; LUCAS, Victor; SILVANO, Zarur. Pesquisa operacional utilizada na maximização do processo de fabricação de discos de algodão em uma empresa do ramo de algodão hidrófilo.

SILVA Elves Magdo, CARVALHO Roberval Rymer da Silva, JÚNIOR Vladas. Pesquisa Operacional Aplicada na Maximização de Receita em uma Academia de Ginástica. Simpósio de Excelência em Gestão e Tecnologia. Disponível emhttps://www.aedb.br/seget/arquivos/artigos08/194_PO\%20APLICADA\%20MAX\%20RECEITA\%2 OACADEMIA.pdf Acesso 14/11/2018

SLACK, N.; CHAMBERS, S.; HARLAND, C. et al. Administração da produção. São Paulo: Atlas, 1996.

TARDIN et al (2013) apud GIL (1999). XXXIII Encontro Nacional de Engenharia de Produção, Enegep. 2013. 